\title{
BIBECHANA
}

A Multidisciplinary Journal of Science, Technology and Mathematics

ISSN 2091-0762 (online)

Journal homepage: http://nepjol.info/index.php/BIBECHANA

\section{Asymmetric mass-loss from the white dwarf WD 0253+209: Secret revealed}

\author{
Binil Aryal \\ Central Department of Physics, Tribhuvan University, Kirtipur, Nepal \\ e-mail: binil.aryal@uibk.ac.at \\ Article history: Received 25 May, 2011; Accepted 8 June, 2011
}

\begin{abstract}
Dust structures around the white dwarf WD 0253+209 is studied in 100 and 60 micron infrared image. These images are received from Infrared Astronomical Satellite Survey (IRAS Survey). The post Asymptotic Giant Branch (AGB) emission of the white dwarf's precursors' wind and the ambient interstellar matter is studied. The distribution of the relative flux density is studied and analyzed in the context of the dust color temperature, mass loading trend and the amount of total mass deposited due the interaction in the interstellar medium. The twisted curved emission structure at 100 micron in the region of interest is probably due to the interaction between the ambient interstellar medium and the He-flashes of the parent planetary nebula of the central white dwarf WD $0253+209$. The total mass of the filamentary arc is found to be $\sim 5$ solar masses, as predicted. The mass loss rate of the post AGB star goes up to $10^{-5}$ solar masses per year. It is concluded that the first He-flash occurred at least $\sim 2500$ years ago.
\end{abstract}

Keywords: white dwarf; interstellar medium; flux density; interstellar dust; mass of the gas

\section{Introduction}

When a star exhausts the supply of hydrogen in its core, the core contracts and its temperature increases, causing the outer layers of the star to expand and cool. The star's luminosity increases greatly, and it becomes a red giant, following a track leading into the upper-right hand corner of the HR diagram [5,13]. The Asymptotic Giant Branch is the name given to a region of the HertzsprungRussell diagram populated by evolving low to medium-mass stars. During this phase the star swells up to giant proportions to become a red giant again. The star may become as large as one astronomical unit. After the helium shell runs out of fuel, the TP-AGB starts. Now the star derives its energy from fusion of hydrogen in a thin shell, inside of which lies in the now inactive helium shell. However, on periods of 10,000 to 100,000 years the helium shell switches on again, and the hydrogen shells switches of, a process known as a helium shell flash [6].

Towards the end of their lifetime, almost all (95-98\%) stars lose a substantial fraction of their mass on the Asymptotic Giant Branch (AGB) in form of massive winds, which compels them into the Planetary Nebula (PN) phase [13]. The central star of PN is the white dwarf. The massive stellar wind emitted from the AGB star interacts with the ambient interstellar medium. Thus the structure (gas or the dust) around the white dwarf preserve the history as a fossil record of the early and late AGB phase of the star. It is important to understand the shaping process of interstellar clouds as well as the nature of the emission from AGB to PN phase. The white dwarf is suspected to reside within giant dust structures which may represent fossil records of its progenitor's transition from spherically symmetric to bipolar or unipolar mass loss [1,2]. It can be suspected that the white dwarf is not the only one where fossil records of ancient mass loss in its neighborhood as well as signs of the resulting shaping 
Binil Aryal / BIBECHANA 8 (2012) 1-7 : BMHSS, p.2

can be traced. Besides being ideal laboratories for the study of various astrophysical processes prevailing in highly excited dilute nebulae, $\mathrm{PNe}$ and their progenitors are key objects for the understanding of the evolution of stars. In this connection, the transformation from spherically symmetric AGB winds to non-spherical PNe represents one of the most enduring problems of stellar astrophysics (e.g. Aryal, Rajbahak \& Weinberger, 2009; Balick, 2002) [3,4].

In this work we present a detailed study of dust structures around white dwarf WD 0253+209 in order to understand the fossil records of its evolution during AGB phase. For this, the variation of flux density along the maxima, dust color temperature profile of the region, mass of the gas in the nods and the possible direction of the mass loading have been extensively examined.

\section{Methods}

We have carried out following steps to study the dust structure around the white dwarf WD $0253+209$.

1. We searched 100 micron dust structure around hot white dwarfs.

2. The past history of the evolution of white dwarfs is studied by carefully examining the dust structure around the white dwarf.

3. The interaction between the stellar wind in the post AGB phase is studied.

4. We intended to study the mass loading phenomena around the investigated white dwarf. We adopt the following methods to find the dust color temperature of the region of interest and the mass of the gas in the selected region around the white dwarf.

\subsection{Temperature Estimation}

We followed the method developed by Schnee et al. (2005) to calculate the dust color temperature from the IRAS 60 micron and 100 micron flux densities [14]. The temperature is determined by the ratio of the 60 micron and 100 micron flux densities. The dust temperature $T_{d}$ in each pixel of a FIR image can be obtained by assuming that the dust in a single beam is isothermal and that the observed ratio of 60 micron to 100 micron emission is due to black body radiation from dust grains at $T_{d}$, modified by a power law emissivity spectral index. The flux density of emission at a wavelength $v$, is given by

$$
F_{i}=\left[\frac{2 h c}{\lambda_{i}^{2}\left(e^{\left.\frac{h c}{\lambda_{i} K T_{d}}-1\right)}\right.}\right] N_{d} \alpha \lambda_{i}^{-\beta} \Omega \Omega_{l}
$$

where $N_{d}$ represents column density of dust grains, $\alpha$ is a constant that relates the flux to the optical depth of the dust, $\beta$ is the emissivity spectral index, and $\Omega_{\mathrm{l}}$ is the solid angle subtended at $\lambda_{\mathrm{i}}$ by the detector. Following Dupac et al. (2003), we use the equation [8]

$$
\beta=\frac{1}{\delta+\omega T_{d}}
$$

to describe the observed inverse relationship between temperature and emissivity spectral index. With the assumptions that the dust emission is optically thin at 60 micron and 100 micron and that $\Omega_{\mathrm{w}} \approx$ $\Omega_{100}$ (true for IRAS image), we can write the ratio, R, of the flux densities at 60 micron and 100 micron as

$$
R=0.6^{-(3+\beta)} \frac{e^{\frac{141}{T_{d}}} 1}{e^{\frac{24 D}{T_{d}}-1}}
$$

Once the appropriate value of $\beta$ is known, one can use Eq. (3) to derive $T_{d \cdot}$. The value of $\beta$ depends on such dust grain properties as composition, size, and compactness. For reference, a pure blackbody would have $\beta=0$, the amorphous layer-lattice matter has $\beta \sim 1$, and the metals and crystalline dielectrics have $\beta \sim 2$. For a smaller value of $\mathrm{Td}, 1$ can be dropped from both numerator and denominator of Eq. (3) and it takes the form 


$$
R=0.6^{-(3+\beta)} \frac{e^{\frac{144}{T_{d}}}}{e^{\frac{24 d}{T_{d}}}}
$$

Taking natural logarithm on both sides of Eq. (4) we find the expression for the temperature as

$$
T_{d}=\frac{-95}{\ln \left\{R \times 0.6^{-(3+\not)}\right\}}
$$

where $\mathrm{R}$ is given by

$$
R-\frac{F(60 \text { micron })}{F(100 \text { micron })}
$$

\subsection{Mass Estimation}

The dust masses are estimated from the infrared flux densities at 60 micron and 100 micron, following the analysis of Meaburn et al. 2000; Young et al., 1989, and Donofrio et al., 1999 (equation 7) [12, 15, 7]. The infrared flux can be measured from IRAS Sky-View images and images from the Groningen using both ALADIN2.5 and FITSVIEW2.0.1 software. The background subtraction is done by the averaging the background surrounding the object and multiplying by the number of pixels containing the object. The black body intensity can be calculated using the basic expression as given in equation (8). The resulting dust mass depends on the physical and chemical properties of the dust grains, the adopted dust temperature and the distance to the object

$$
m_{\text {dust }}=\frac{4}{3} \frac{\alpha \rho}{Q_{v}}\left[\frac{S_{v} D^{2}}{B(v, T)}\right]
$$

where, $\alpha=$ Weighted grain size, $\rho=$ Grain density, $Q_{v}=$ Grain emissivity, $\mathbf{S}_{v}=$ flux density. The Planck's function is a well known function, given by this equation,

$$
B(v, T)=\frac{2 h v^{3}}{c^{2}}\left[\frac{1}{\exp \left(\frac{h v}{N T}\right)-1}\right]
$$

It is clear from the expression (8) that the value of Planck Function $[B(v, T)]$ for longer wavelength is higher than that of the shorter wavelength. Consequently, the range of $B(v, T)$ for fixed temperature goes narrower if wavelength of the images increases.

\subsection{Region of Interest}

We planned to study the region as shown in Fig. 1a. This region has one white dwarf, namely WD $0253+209$ (see plus mark in Fig. 1). The position of this white dwarf is R.A. $(J 2000)=02^{\mathrm{h}} 55^{\mathrm{m}} 4.37^{\mathrm{s}}$ and Dec. $(\mathrm{J} 2000)=+21^{\circ} 06^{\prime}, 08^{\prime \prime}$.

\section{Result and Discussion}

First we present the results concerning the interaction between the stellar wind emitted from the post AGB phase of the white dwarf with the surrounding interstellar medium. The study of relative flux density helps us to know about the inner dynamics of the region. In addition, this study is useful to estimate the dust color temperature and the mass of the dust in the selected region. After getting information regarding the possible energy needed to make the structure, we calculate/estimate whether this amount of energy can be supplied by the white dwarfs or not. 


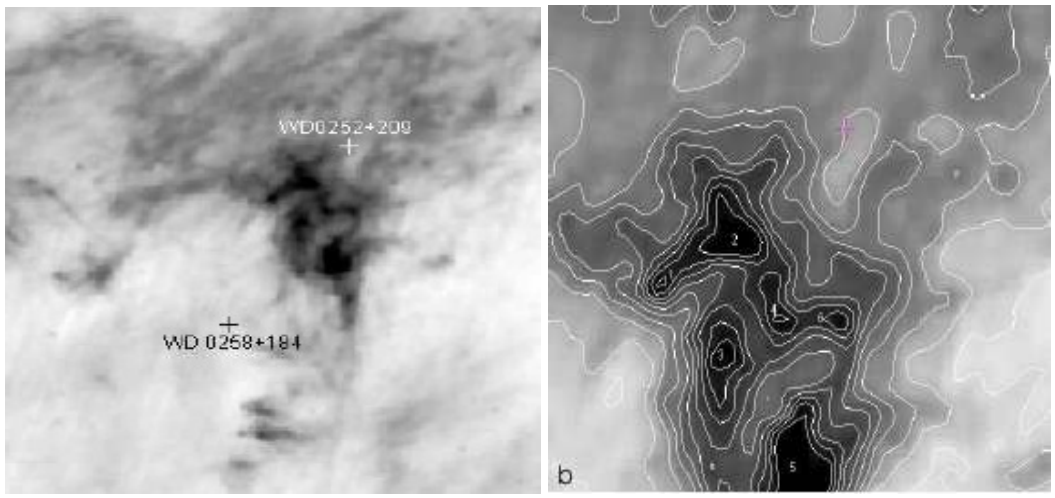

Fig. 1: (a) 100 micron IRAS image of the region of interest. The symbol 't' represents the position of white dwarf WD 0253+209. (b) Contour maps showing the rounded filamentary nodes around the white dwarf.

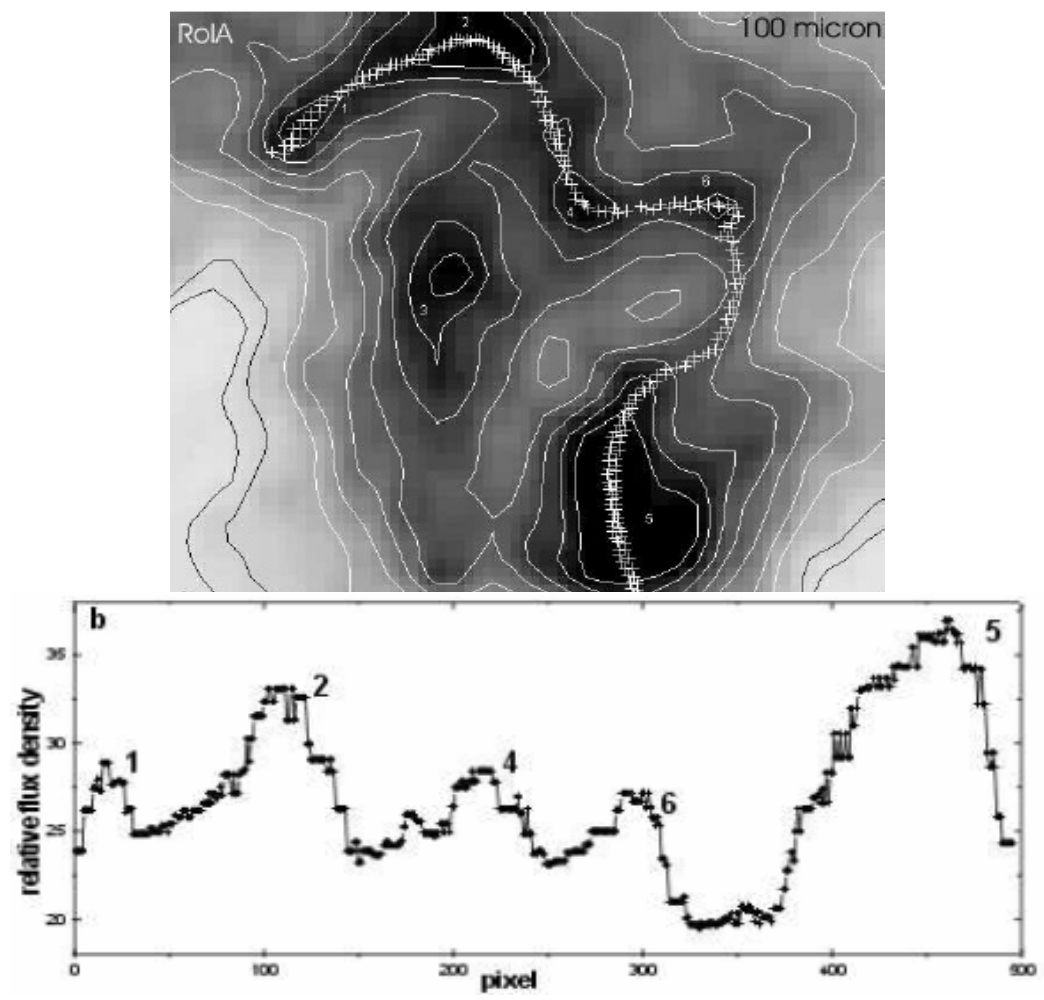

Fig. 2: (a) Region of interest (ROI) showing line joining the maxima of the nodes as shown in Fig. $1 \mathrm{~b}$. The variation of relative flux density is plotted along the path, starting from northern maxima, i.e., ' 1 ' to the southern maxima i.e., ' 5 '.

The studied region has one white dwarf, namely WD $0253+209$. The position of this white dwarf is R.A. $(\mathrm{J} 2000)=02^{\mathrm{h}} 55^{\mathrm{m}} 4.37^{\mathrm{s}}$ and Dec. $(\mathrm{J} 2000)=+21^{\circ} 06^{\prime}, 08^{\prime \prime} .100$ micron IRAS image centered at R.A. $(\mathrm{J} 2000)=02^{\mathrm{h}} 55^{\mathrm{m}} 1.8^{\mathrm{s}}$, Dec. $(\mathrm{J} 2000)=21^{\circ} 05^{\prime} 32^{\prime \prime}$ is shown in Fig. 1a. The position of the white dwarf is shown by the symbol ' + '. In the northern part of white dwarf, grey shaded filamentary arc can be seen. The contour map of the image presented in Fig. 1b shows five maxima (represented by 1, 2, 4,6 and 5 ) from top to bottom. These maxima have 2.5 times greater values of relative flux density 
than that of the background. The minimum and maximum relative flux density is found at maxima ' 2 ' and ' 5 '.

Fig. 2 a shows the path joining all the maxima from ' 1 ' to ' 5 '. The path goes through $1 \rightarrow 2 \rightarrow 4 \rightarrow 6 \rightarrow$ 5 from top to bottom. The value of flux density is measured at all pixels on the path. Fig. $2 b$ shows the variation of relative flux. The maximum relative flux density at ' 2 ' and ' 5 ' are nearly equal. Maxima ' 4 ' showed less flux than that of ' 2 ' and ' 5 '. Interestingly it is found that the path joining the maxima $1 \rightarrow 2$ $\rightarrow 4 \rightarrow 6 \rightarrow 5$ are point of twisted filamentary arc. It is noticed that the mass at ' 1 ', ' 2 ' and ' 4 ' are growing day by day in equal rate. It is also found that the separation between all the maxima is equal.

Star with initial masses between ' 1 ' and ' 9 ' solar masses that end up as white dwarf must lose a substantial fraction of their mass before they leave asymptotic giant branch (AGB). Most of the mass loss occurs near the end of AGB lifetime. These losses of mass are appear in the form of nodes around the white dwarf. As time passes, mass is found to be deposited rapidly around the white dwarf. To study the mass loading on the maxima, we joined the maxima of the infrared clouds and the white dwarf (see Fig. 3a). The variation of relative flux density along the path helps us to understand the mass loss pattern.

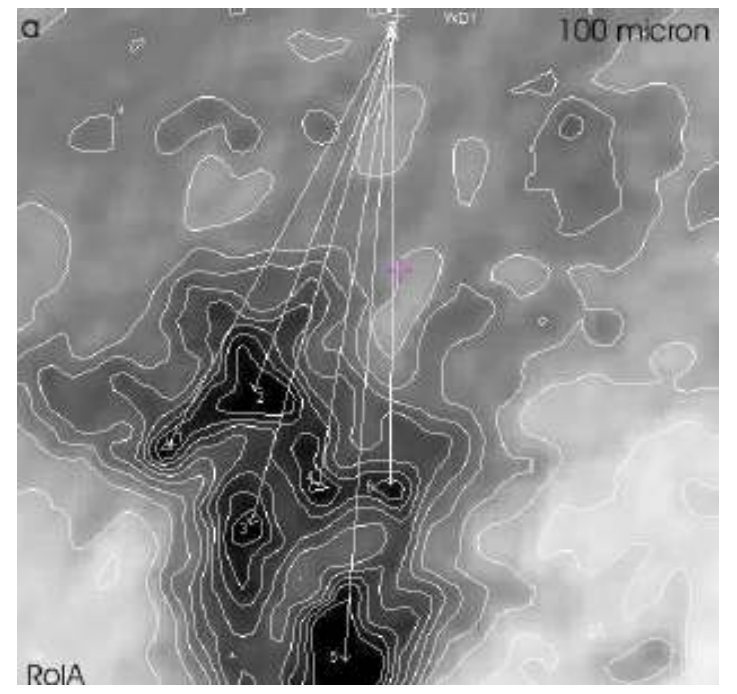

Fig. 3: Variation of relative flux density along straight line joining the maxima $(1,2,3,4,5$ and 6$)$ and the white dwarf WD 0253+209.

To calculate the dust color temperature we used the 100 micron and 60 micron IRAS images in ALADIN2.5 software. The maximum temperature in the maxima ' 1 ' found to be $18.5 \pm 1.5 \mathrm{~K}$. This temperature is the background corrected. The maximum value of dust color temperature of the maxima '2', '3', '4', '5' and '6' are estimated as $17.5 \pm 0.5 \mathrm{~K}, 18.5 \pm 0.5 \mathrm{~K}, 17.5 \pm 0.5 \mathrm{~K}, 18.5 \pm 1.5 \mathrm{~K}$ and $18.0 \pm 1.0 \mathrm{~K}$ respectively. The error in the temperature is calculated by estimating the minimum temperature of that region. Thus, the maxima ' 1 ', ' 3 ' and ' 5 ' is found to be hotter than other maxima. Interestingly, the region ' 2 ' is found to be colder than that of the region ' 1 ', ' 3 ' and ' 5 '. It should be noted that the paths $1 \rightarrow 2 \rightarrow 4 \rightarrow 6 \rightarrow 5$ shows a similar temperature fluctuation, suggesting a close connection between the paths (Fig. 4).

The mass of the dust is estimated using Henning et al. (Eq. 7). The value of their respective temperature is used in order to calculate the value of the Planck function (Eq. 8). We used 100 micron IRAS image for the mass estimation. The region ' 5 ' is obviously the massive region. It has a mass of $(1.670 \pm 0.940) \mathrm{M}_{\text {sun. }}$. The second and third massive region is found to be region ' 3 ' and region ' 2 ', where $(1.210 \pm 0.250) \mathrm{M}_{\text {sun }}$ and $(1.200 \pm 0.280) \mathrm{M}_{\text {sun }}$ are estimated. The least massive maxima in the RolA value and the error are estimated using the range of flux density in each maxima region. All together, RolA has a mass of $(4.732 \pm 0.940) \mathrm{M}_{\text {sun. }}$. Now the question is, this amount of mass can be 


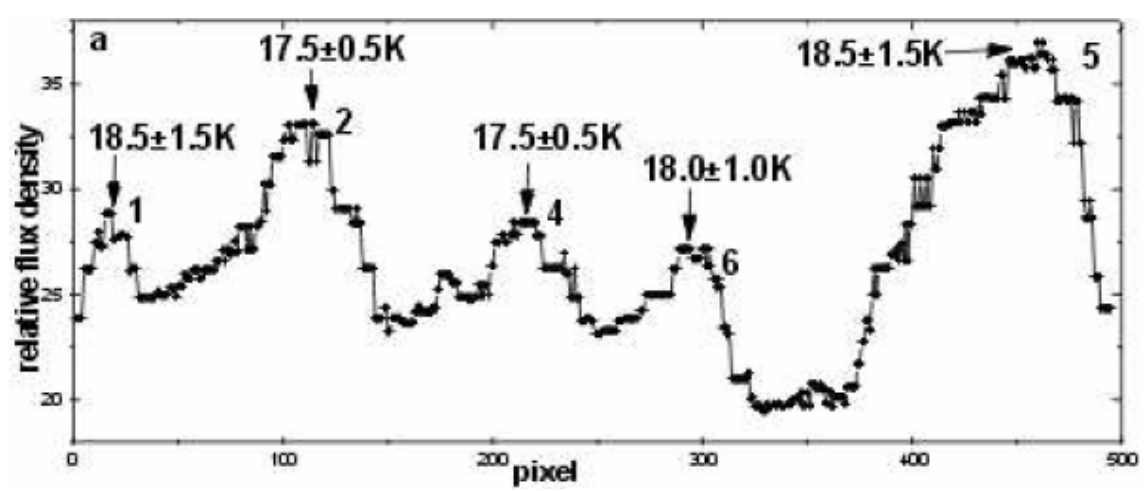

Fig. 4: The variation of relative flux density is plotted along the path which connects the maxima $1 \rightarrow 2$ $\rightarrow 4 \rightarrow 6 \rightarrow 5$. The maximum temperature at the maxima is shown.

contributed by our white dwarfs or not? Obviously not! Because white dwarf is the remnant of 1.440 $M_{\text {sun }}$ star. However, the deposited masses in the ISM region is need to be added here. We check whether our white dwarf can displace and load this amount of estimated mass during their evolution or not.

In the optical (red) wavelength, the surface brightness of this white dwarf is extremely low. The spectral index of this white dwarf in V band is 17.83 (McSion \& Cook 2003). So, this white dwarf is relatively colder. In the catalog, this white dwarf is classified as DA, means it has only Balmer lines, no $\mathrm{He}$ I or metals present. The white dwarf (WD 0252+209) which is at the head of the emission structure is colder (i.e., older) than that of the white dwarf (WD 0258+184) at the tail or bottom of the structure. The difference in the spectral index in $\mathrm{V}$ band is, 17.83-15.30 $=2.53$. This difference leads age difference of $\sim 2500$ years. This difference is significant in the context of the total mass of the emission region of the structure, i.e., $\sim 5 \mathrm{M}_{\text {sun. }}$. It is found that the mass-loading is active in the lower part but the contribution of upper white dwarf is dominating in the context of the amount of mass deposition. Thus, the structure RolA is formed due to the interaction between the post AGB winds of the white dwarf. However, this interaction is found to be active in the lower region.

We conclude that the twisted curved structure in the region of interest is formed probably due to the interaction between the ambient interstellar medium and the He flashes of the parent planetary nebula of the white dwarf WD 1003-441.

\section{Conclusion}

We studied the 100 micron dust structures around white dwarf WD 1003-441. The post Asymptotic Giant Branch (AGB) emission of white dwarf's precursors' wind and the ambient interstellar matter is thoroughly studied. For this, distribution of the relative flux density of each pixel of the images are analyzed in the context of the dust color temperature, mass loading trend and the amount of total mass deposited due the interaction in the interstellar medium. The 100 micron far infrared image is provided by the Groningen server of Infrared Astronomical Satellite (IRAS) survey. The spectral index of the white dwarf was taken from McSion \& Cook (2003). These images were processed in the image reduction software ALADIN2.5. The calculations for the dust color temperature (Schnee et al. 2005 \& Dupac et al., 2003) and the mass (Young 1989) were carried out using 100 micron IRAS image.

The white dwarf (WD 0252+209) which is at the head of the emission structure is found to be colder (i.e., older) than that of the white dwarf (WD 0258+184) at the tail or bottom of the emission structure (region of interest A). The difference in the spectral index in V (2.53) leads age difference of $\sim 2500$ years. This difference is noticeable because total mass of the emission region of the structure is not more than 5 solar masses. It can be concluded that the process of mass-loading is active in the lower part but the contribution of northern white dwarf (WD 0253+209) is dominating. The maximum and minimum dust color temperature of the dust structure is found to be 20.0 and $17.0 \mathrm{~K}$, respectively. It can be concluded that the structure is formed due to the interaction between the post AGB winds of the white dwarf. 


\section{Acknowledgements}

B. Aryal is grateful to the University of Innsbruck as well as to its Institute of Astro- and Particle Physics for financial support for a research stay during October to December 2010. We acknowledge Prof. Ronald Weinberger of Innsbruck University for his important suggestion. This research has made use of NASA/IPAC Extragalactic Database (NED) which is operated by the Jet Propulsion Laboratory, California Institute of Technology, under contract with the National Aeronautics and Space Administration.

\section{References}

[1] B. Aryal, R. Weinberger, Journal Astronomy \& Astrophysics 245 (2006) 306.

[2] B. Aryal, C. Rajbahak, R. Weinberger, Monthly Notice of Royal Astronomical Society 402 (2010) 1307.

[3] B. Aryal, C. Rajbahak, R. Weinberger, Astrophysics \& Space Science Journal 223 (2009) 223.

[4] B. Balick, Journal Astronomy \& Astrophysics 282 (2002) 143.

[5] R. D. Blandford, M. J. Rees, Monthly Notice of Royal Astronomical Society 169 (1974) 395.

[6] D. P. Cox, Journal Astronomy \& Astrophysics 43 (2005) 237.

[7] R. Donofrio, et.al., Journal Astronomy \& Astrophysics Supp. 105 (1999) 12.

[8] X. Dupac et al., Publication Astron. Society Asia Pacific 115 (2003) 965.

[9] http: //simbad.u-strasbg.fr (Official web page of SIMBAD Database)

[10] http: //skyview.gsfc.nasa.gov (Official web page of SKYVIEW Survey)

[11] M. McSion, S.M. Cook, Catalog of WD, Groningon (2003), The Netherland

[12] D. Meaburn, et.al., Journal Astronomy \& Astrophysics 215 (2000) 903.

[13] M. J. Rees, Annu. Rev. Astron. \& Astrophysics 22 (1984) 471.

[14] S. L. Schnee, A. R. Naomi, A. G. Alyssa et al., Astrophysical Journal 634 (2005) 442.

[15] W. Young, Astrophysical Journal 109 (1989) 725. 